\title{
DN1p or the "Fluffy" Cerberus of Clock Outputs
}

\author{
Angélique Lamaze* and Ralf Stanewsky \\ Institut für Neuro und Verhaltensbiologie, Westfälische Wilhelms University, Münster, Germany
}

Drosophila melanogaster is a powerful genetic model to study the circadian clock. Recently, three drosophilists received the Nobel Prize for their intensive past and current work on the molecular clockwork (Nobel Prize 2017). The Drosophila brain clock is composed of about 150 clock neurons distributed along the lateral and dorsal regions of the protocerebrum. These clock neurons control the timing of locomotor behaviors. In standard light-dark (LD) conditions $\left(12-12 \mathrm{~h}\right.$ and constant $\left.25^{\circ} \mathrm{C}\right)$, flies present a bimodal locomotor activity pattern controlled by the clock. Flies increase their movement just before the light-transitions, and these behaviors are therefore defined as anticipatory. Two neuronal oscillators control the morning and evening anticipation. Knowing that the molecular clock cycles in phase in all clock neurons in the brain in LD, how can we explain the presence of two behavioral activity peaks separated by $12 \mathrm{~h}$ ? According to one model, the molecular clock cycles in phase in all clock neurons, but the neuronal activity cycles with a distinct phase in the morning and evening oscillators. An alternative model takes the environmental condition into consideration.

OPEN ACCESS

Edited by:

Sylvia Anton,

Institut National de la Recherche

Agronomique (INRA), France

Reviewed by:

Kenji Tomioka,

Okayama University, Japan Frank Klaus Schubert, University of Wuerzburg, Germany

*Correspondence:

Angélique Lamaze

alamaze@uni-muenster.de angie0203@hotmail.com

Specialty section: This article was submitted to Invertebrate Physiology, a section of the journal Frontiers in Physiology

Received: 19 September 2019 Accepted: 05 December 2019

Published: 08 January 2020

Citation:

Lamaze $A$ and Stanewsky $R$ (2020) DN1p or the "Fluffy" Cerberus of Clock Outputs.

Front. Physiol. 10:1540. doi: 10.3389/fphys.2019.01540
One group of clock neurons, the dorso-posterior clock neurons DN1p, drive two peaks of locomotor activity in LD even though their neuronal activity cycles with the same phase (late night/early morning). Interestingly, the locomotor outputs they control differ in their sensitivity to light and temperature. Hence, they must drive outputs to different neuropil regions in the brain, which also receive different inputs. Since 2010 and the presentation of the first specific DN1p manipulations, many studies have been performed to understand the role of this group of neurons in controlling locomotor behaviors. Hence, we review what we know about this heterogeneous group of clock neurons and discuss the second model to explain how clock neurons that oscillate with the same phase can drive behaviors at different times of the day.

Keywords: Drosophila melanogaster, circadian clock, DN1p, locomotor activity, temperature response

\section{INTRODUCTION}

The fundamental function of the circadian clock is to synchronize the organism with its ecological niche. The circadian period is genetically determined (Konopka and Benzer, 1971) and therefore, does not depend on the environment, like the ambient temperature for example. However, $24 \mathrm{~h}$ oscillations of environmental parameters, such as daily light and temperature cycles (TC), synchronize the clock. Also, clock-controlled behavior is phased (time of occurrence within the $24 \mathrm{~h}$ period) based on the current environmental status. An individual's locomotor activity pattern therefore depends on complex neuronal networks, integrating both environmental inputs and genetically encoded endogenous time information. Drosophila melanogaster is a reference model to study the circadian clock not only for its tremendous genetic advantages (Nobel prizes 2017), but it is also easy to tightly control the environment when it comes to study its locomotor behavior. When 
isolated in a small glass tube, the "dew lover" Drosophila displays a highly plastic locomotor behavior that changes with light and temperature. In standard light-dark 12-12 h (LD) cycles and constant mild temperature $\left(22-25^{\circ} \mathrm{C}\right)$, male flies present two peaks of locomotor activity. In the late night, flies start to wake up in a synchronous manner and increase their locomotor activity. The light transition induces a startle response, after which the activity decays and male flies start their siesta. Then, in the late afternoon, they again increase their locomotion in a synchronous manner "anticipating" the lights-off transition. Because these activities occur several hours before the lighttransitions, they have been defined as anticipatory. However, at cooler temperatures $\left(18^{\circ} \mathrm{C}\right)$, the morning anticipation is strongly dampened or delayed, while the evening one is advanced compared to $25^{\circ} \mathrm{C}$. Inversely, at warmer temperatures $\left(29^{\circ} \mathrm{C}\right)$, the amplitude of the morning anticipation increases and advances while the evening anticipation delays (Majercak et al., 1999).

In constant light and temperature (LL), fruit flies are arrhythmic. This is due to the constitutive degradation of the clock protein TIMELESS (TIM), mediated by the circadian photoreceptor cryptochrome (cry) (Stanewsky et al., 1998). However, flies can entrain to TC in LL (LLTC 25-16 ${ }^{\circ} \mathrm{C}$ ) (Glaser and Stanewsky, 2005; Yoshii et al., 2005). Nonetheless, the behavior observed when only light alternates is different from the one observed in LLTC, which is again different from the one observed in the same TC but in constant darkness (DD) (Gentile et al., 2013). In LLTC, we observe a unique anticipatory activity peak at the end of the thermophase, while in DDTC this peak shifts toward the beginning of the thermophase.

The Drosophila brain clock is composed of about 150 clock neurons (Figure 1A). In 2004, two labs showed that if a functional clock is restricted to a group of $\mathrm{CRY}^{+}$lateral neurons (LN) expressing the neuropeptide pigment dispersing factor (PDF), this is sufficient to drive the morning anticipation, which therefore was named lateral neurons-morning oscillator (LN$\mathrm{MO})$. In contrast, a clock restricted to LN expressing CRY but not $\mathrm{PDF}$ is sufficient to drive the evening anticipation (Figure 1B), and therefore was defined as lateral neurons-evening oscillator (LN-EO) (Grima et al., 2004; Stoleru et al., 2004).

In 2010, Zhang Y. et al. (2010) showed that a clock in a group of about 12 clock neurons located in the dorsal part of the protocerebrum, the DN1p, is sufficient to drive both morning and evening anticipation, albeit under distinct light and temperature conditions (Figure 1C). It was the first time that a group of clock neurons was found capable of controlling locomotor activity twice a day. By definition, a circadian output is a behavior that occurs every $24 \mathrm{~h}$. Therefore, how do clock neurons with a 24 -h molecular clock cycling in phase, control locomotor activity twice a day? Contrary to the EO and the MO whose neuronal activities cycle at different phase (Liang et al., 2016), the neuronal activity cycles in phase within the DN1p group (Flourakis et al., 2015; Liang et al., 2016). However, there is heterogeneity within this group. Half of them express cry (Benito et al., 2008), not all of them are glutamatergic (Hamasaka et al., 2007; Chatterjee et al., 2018) and a certain proportion of them express the neuropeptide DH31 (Kunst et al., 2014) or allatostatin-C (Diaz et al., 2019). It is still unclear which subgroup of DN1p controls the morning activity and which one controls the evening one, although the glutamatergic and $\mathrm{CRY}^{+} \mathrm{DN} 1 \mathrm{p}$ seem to have a predominant role in regulating morning activity (Chatterjee et al., 2018).

To understand how DN1p clock neurons contribute to the complex circadian regulation of locomotor behavior, we review what is known about this intriguing cluster of neurons and will try to emphasize how their comprehension can help us to understand how neurons integrate and relay multiple inputs.

\section{DN1p: A NON-AUTONOMOUS "CIRCADIAN" OSCILLATOR}

By definition, circadian clocks tick autonomously. In the absence of environmental input (DD $25^{\circ} \mathrm{C}$ ), organisms maintain their rhythm of about $24 \mathrm{~h}$, they free run. In the Drosophila brain, clock proteins maintain their rhythms for days in most of the clock neurons including the DN1p, while most of the peripheral clocks stop their oscillations after a few days in constant condition (Veleri et al., 2003). However, in the absence of PDF or in the absence of PDF cells, the DN1p loose their oscillations in DD very quickly (Veleri et al., 2003; Klarsfeld et al., 2004; Yoshii et al., 2009), suggesting a dependency of these neurons on the LN-MO. Indeed, when the pace of the molecular clock is genetically modified in the LN-MO, clock proteins and tim mRNA oscillate in the DN1p following the pace of the LNMO (Stoleru et al., 2005; Chatterjee et al., 2018). How do PDF neurons dictate the rhythm to the DN1p? When PDF links to its receptor (PDFR, a G protein-coupled receptor), this leads to an increase of cAMP (Shafer et al., 2008), which activates protein kinase A (PKA). Interestingly, Seluzicki et al. (2014) have observed that a rescue of PER oscillations specifically in the LN-MO is sufficient to drive TIM oscillations in DN1p. They suggested that PDF regulates TIM levels via a PKA signaling pathway (Seluzicki et al., 2014). However, we do not know whether TIM is directly phosphorylated and stabilized by PKA in a PDF-dependent manner. Nonetheless, this hypothesis provides a nice model of the DN1p pace-regulation by the LNMO (Figure 2). Furthermore, in the absence of both CRY and PDF, PER expression in the DN1p becomes arrhythmic even in LD (Cusumano et al., 2009), reinforcing this dependency toward the LN-MO.

Hence, although they express the molecular circadian machinery, the DN1p miss an unknown element providing autonomy, potentially a factor that regulates TIM oscillations in a CRY and PDF independent manner. Like peripheral clocks, the DN1p are not autonomous and only maintain their rhythm in DD thanks to the $\mathrm{PDF}^{+}$pacemaker neurons.

\section{THE ROLE OF DN1p IN REGULATING LOCOMOTOR BEHAVIOR IN CONSTANT DARKNESS}

A rhythmic circadian output is defined by its period and the phase of its peak and trough. The period of a circadian output is genetically determined. However, the phase is determined by 

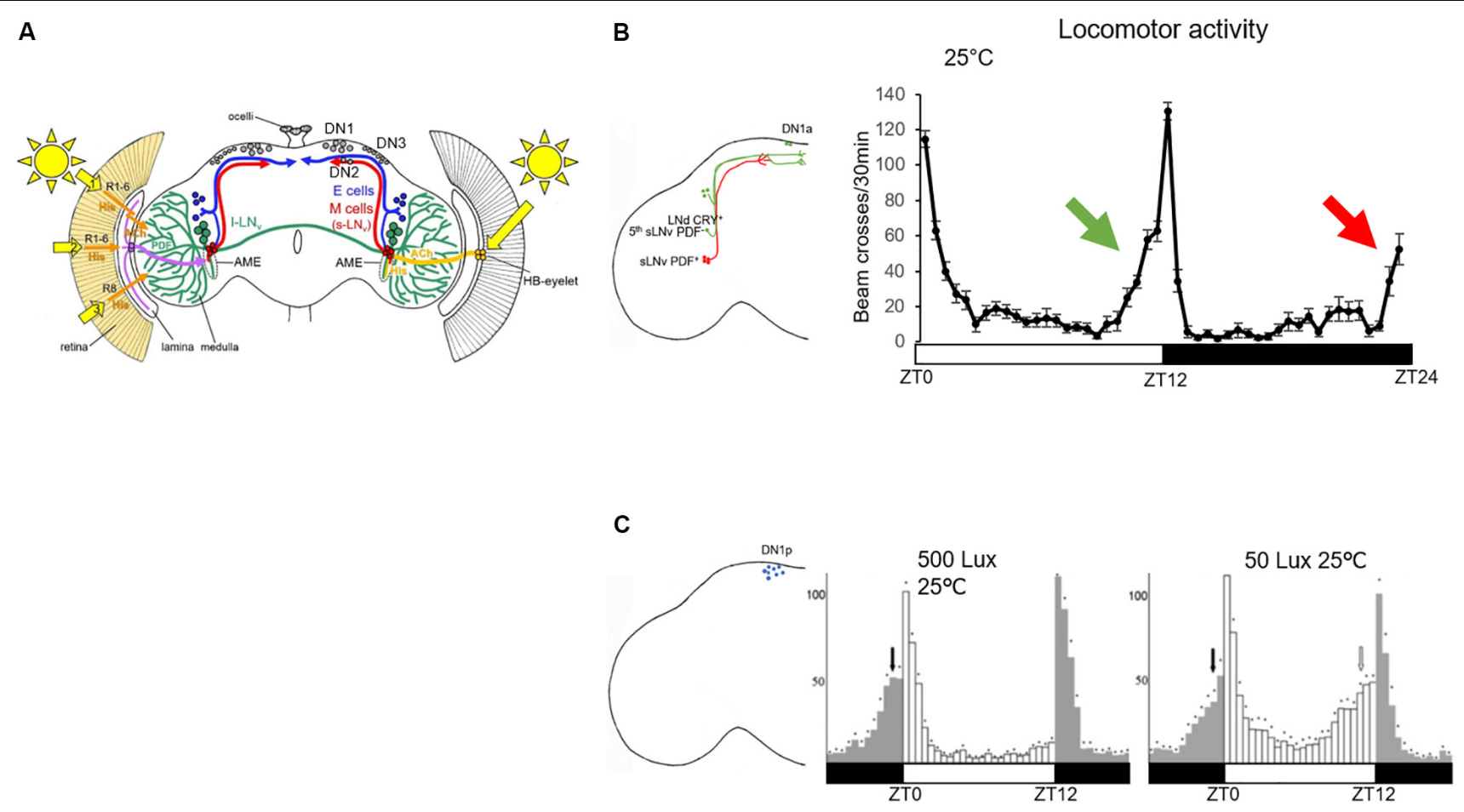

FIGURE 1 | Role of clock neurons in controlling the locomotor behavior in standard LD conditions. (A) Clock neurons in the Drosophila brain. Figure taken from Helfrich-Förster (2019). Numbers 1-3 indicate the three putative opsin-based light input pathways to the clock neurons in addition to the HB-eyelet input (Helfrich-Förster, 2019). The I-LNv are represented in green, the LN-EO (E cells) in blue and the LN-MO (M cells) in red. The dorsal neurons (DN1-DN3) are colored in gray. Their projection pattern is not shown. The axonal projections of the lateral neurons are located in the dorso-posterior area of the brain (except for the ILNV, which project to the ipsi- and contralateral medulla). Most of the clock neuron dendritic projections are in the accessory medulla (AME) (Li et al., 2018). (B) Left: schematic representation of the LN-MO and LN-EO only, with their output projections. Right: locomotor activity trace of a group of 31 control flies (iso31) in standard $\mathrm{LD}$ and constant $25^{\circ} \mathrm{C}$. The green arrow indicates the control of the evening anticipation by the EO (green cells in the brain) and the red arrow the morning anticipation by the $\mathrm{MO}$ (red cells in the brain). (C) Left: schematic representation of DN1p cell bodies in the brain; right: locomotor activity traces of per $^{0}$, w; Clk4.1M-Gal4/UAS-per16 (functional clock only in the DN1p) taken from Zhang Y. et al. (2010) in two different light conditions. White bars represents $12 \mathrm{~h}$ of light, dark bars $12 \mathrm{~h}$ of darkness.

the interaction of the animal with its environment. In DD and constant temperature, the main peak of locomotor activity occurs in the subjective evening. The rhythm of this activity pattern is driven by the $\mathrm{PDF}^{+}$neurons (Grima et al., 2004; Stoleru et al., 2004; Figure 3A). Interestingly, when a functional clock is restricted to these neurons, the flies remain rhythmic but their peak of activity shifts toward the subjective morning (Grima et al., 2004). Recently, Chatterjee et al. (2018) were able to change the phase of the DD locomotor activity without affecting the period by genetically modulating the speed of the DN1p clock: phase advanced when the clock was sped up and delayed when the pace was slowed down. While the PDF neurons determine the pace, the DN1p determine the phase. Therefore, we can propose that in the absence of light and temperature oscillations, the endogenous period of the locomotor rhythm is provided by the $\mathrm{PDF}^{+}$neurons and the phase by the non-autonomous DN1p oscillator (Figure 3A). Hence, it seems logical to propose that the DN1p are downstream of the PDF neurons.

The DD locomotor rhythm of $p d f$ mutant flies, or flies lacking its receptor $\left(p d f r^{-}\right)$, is strongly dampened (Renn et al., 1999; Hyun et al., 2005). Interestingly, the rhythm strength can partially be restored when PDF reception is rescued only in DN1p neurons (Zhang L. et al., 2010). Nonetheless, flies are still rhythmic in DD when DN1p output is inhibited using the expression of tetanus toxin (Guo et al., 2016). Even more surprisingly, gl60j mutants that lack DN1p neurons are still rhythmic in DD (HelfrichFörster et al., 2001; Klarsfeld et al., 2004), hence questioning a direct influence of the DN1p on DD rhythmicity.

Looking for downstream neurons important for DD rhythmicity, Cavanaugh et al. (2014) screened for neuronal drivers that lead to arrhythmic locomotor behavior when activated using the thermoreceptor dTrpA1. They identified 10 driver lines with the commonality of showing expression in neuroendocrine cells in the pars intercerebralis (PI), the fly functional homolog of the mammalian hypothalamus. They focused on a line (kurs58-Gal4) that is expressed in 16-18 cells in the PI. After a transcriptomic analysis of individual kurs58 PI cells, they found that some of these neuroendocrine cells express the neuropeptide DH44 (six cells). DH44 is a diuretic hormone, the Drosophila homolog of the stress hormone corticotrophin releasing factor (CRF) (Cabrero et al., 2002) and downregulation of DH44 expression using a pan neuronal 


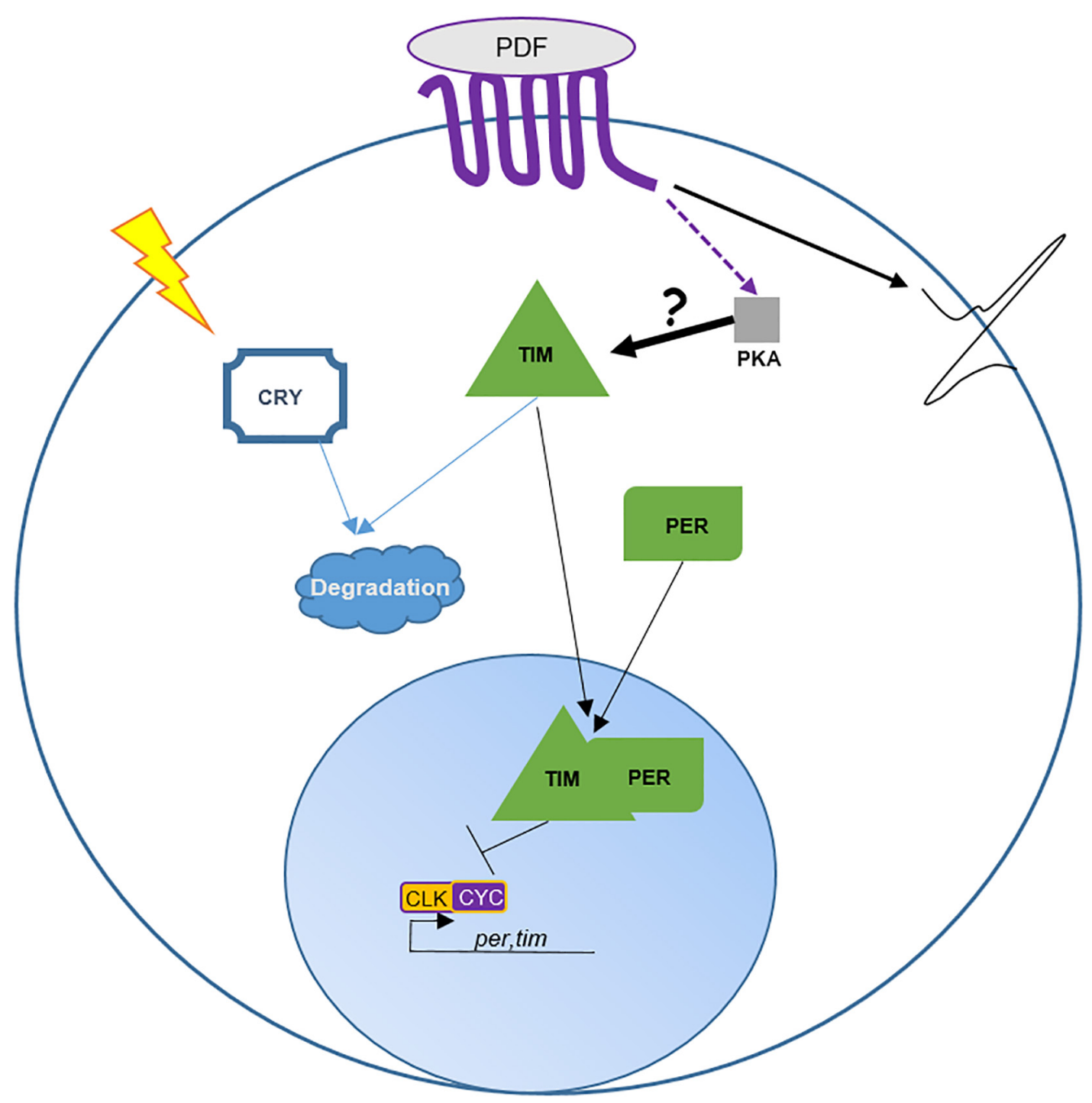

FIGURE 2 | Role of PDF in DN1p entrainment and neuronal activity. This cartoon is based on Seluzicki et al. (2014). The transcription factors CLOCK (CLK) and CYCLE (CYC) form a heterodimer promoting the expression of period (per) and timeless (tim). PER and TIM proteins undergo various post-transcriptional and post-translational modifications delaying their nuclear translocation and inhibition of their own expression by binding to CLK/CYC (Tataroglu and Emery, 2015). Pdfr encodes for a seven transmembrane GPCR protein (Hyun et al., 2005). Activation of PDFR leads to an increase of cAMP in the cytoplasm. cAMP interacts with and activates PKA activity. Directly or indirectly PKA stabilizes TIM in the cytoplasm. In about half of the DN1p TIM is degraded by light via CRY. Interestingly, Seluzicki et al. (2014) observed that PDF promotes DN1p neuronal activity independently of PKA. Hence, the authors suggest that PDF cells entrain the DN1p molecular clock via the control of TIM by PKA and promote DN1p neuronal activity in a PKA-independent manner.

driver strongly decreased rhythmic behavior (Cavanaugh et al., 2014). However, using the same pan neuronal driver and RNAi line, another group was not able to replicate this result (Cavey et al., 2016). Also, specific activation of DH44 neurons with dTrpA1 using the dh $44^{V T}$-gal4 line does not increase the proportion of arrhythmic flies (see Table 1 in Cavanaugh et al., 2014), raising doubts about the real implication of these neurons in regulating DD rhythms. Nonetheless, they do seem to respond to rhythmic input, since their neuronal activity oscillates in DD (Cavey et al., 2016). Interestingly, kurs58-Gal4 is also expressed in sifamide-expressing PI (SIFa) neurons, which are not overlapping with the $\mathrm{DH} 44^{+}$cells (Cavanaugh et al., 2014). The authors observed a decrease of DD rhythmicity in flies deprived of the SIFa neurons (SIFa > reaper) (Cavanaugh et al., 2014) consistent with the observation that pan-neuronal downregulation of SIFa expression strongly decreases rhythmicity in DD (Cavey et al., 2016). Finally, Cavanaugh et al. (2014) found a physical interaction between the DN1p and both the DH44 ${ }^{+}$and the SIFa ${ }^{+}$PI cells. Since kurs58Gal4 is expressed in 16-18 PI cells including the six $\mathrm{DH}_{4} 4^{+}$ and four SIFa neurons, we cannot conclude with certainty the influence of the DH44 neurons on locomotor rhythmicity in 
A

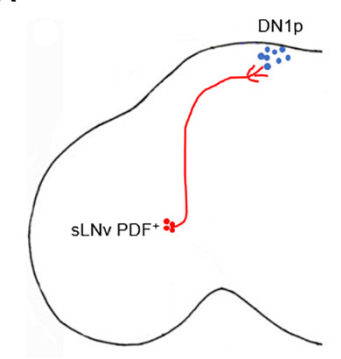

B

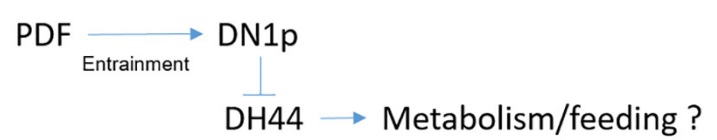

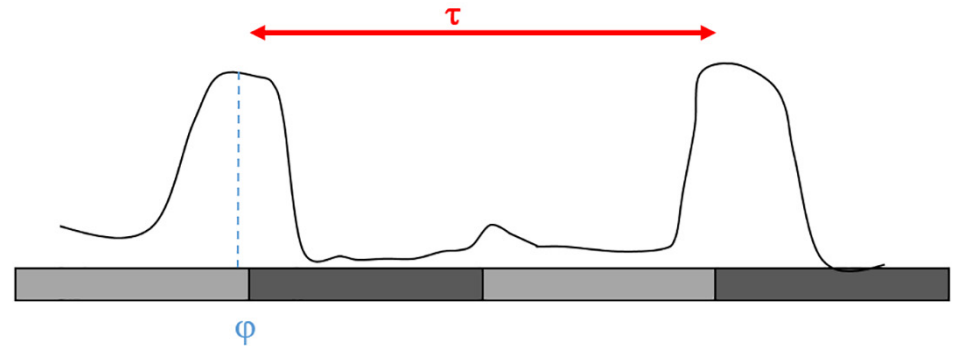

C

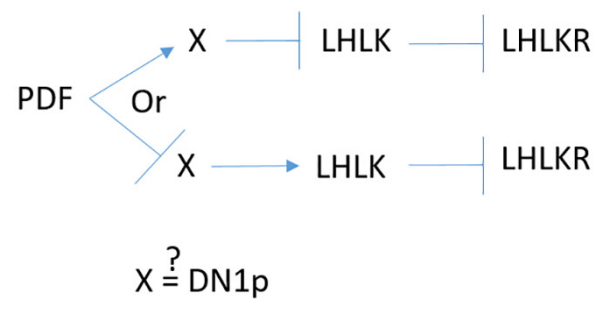

FIGURE 3 | Control of locomotor activity in DD. (A) Left: sketch of sLNv and DN1p in the Drosophila brain. Right: a typical locomotor behavior in DD. Light gray bars represent the subjective day, dark gray bars represent the subjective night. Flies free run with an activity peak at the end of the subjective day. The period $(\tau)$ is defined as the distance between two peaks. $\tau$ is controlled by the sLNv (red), while the phase $(\varphi)$ (when the peak of locomotor activity happens within $\tau$ ) is controlled by the DN1p (blue). (B) Several arguments speak against a role of DH44 in controlling locomotor rhythms in DD (see text). However, the DH44 neurons appear to be rhythmic with a phase opposite to the DN1p (Cavey et al., 2016), and they modulate feeding behavior (Dus et al., 2015). From this, we can postulate that clock neurons influence feeding behavior via rhythmic inhibitory action on the DH44 neurons. (C) In the second model (Cavey et al., 2016), the LHLK neurons are indirectly inhibited by PDF. Hence, either PDF activates an unknown intermediate $(X)$ which in return inhibits the LHLK neurons, or PDF inhibits $X$ which activates LHLK neurons. Since activation of LHLK downstream neurons, LHLKR, changes the phase of the locomotor activity without changing the period of inactivity (Cavey et al., 2016), it is possible that the intermediate are the DN1p neurons.

DD. It would be interesting to further test the potential role of the SIFa neurons.

Later, the same group proposed a potential circuit downstream of the DH44 neurons responsible for DD rhythmicity (King et al., 2017). Flies express two receptors for DH44, DH44R1 and DH44R2 (Hector et al., 2009). While DH44R1 neurons are found in the central nervous system (Dus et al., 2015; King et al., 2017), DH44R2 expressing cells were found in the gut, potentially within the enteroendocrine cells (Dus et al., 2015). King et al. (2017) proposed that a group of DH44R1 neurons localized in the subesophageal zone (SEZ) and expressing the neuropeptide hugin are the downstream target of the DH44 neurons for regulating DD rhythms. However, neither the null mutant for dh44r1 nor the expression of the $d h 44 r 1$ RNAi in hugin ${ }^{+}$neurons lead to arrhythmicity in DD (see Supplementary Table 1 of King et al., 2017). Nonetheless, the authors observed arrhythmic locomotor activity in DD when hugin ${ }^{+}$neurons, along with many others using the R21A07 driver line, are activated (King et al., 2017). DH44 neurons project dendritic arborization to the dorsal region of the SEZ, their cell bodies are highly positive for the presynaptic marker syt-GFP, and they send axonal projections to the gut (Dus et al., 2015). No projections have been observed in the thoracic ganglion, where motor neurons receive inputs from the brain. This projection pattern, along with the absence of arrhythmic behavior in dh44r1 mutants, is therefore not compatible with a role for DH44 neurons in regulating locomotor activity rhythms (Figure 3B).

Another study used a genetic approach to find the circuit downstream of the clock responsible for rhythmic behavior in DD (Cavey et al., 2016). They screened for peptidergic function via expressing RNAis pan-neuronally. From this screen, they found that downregulation of leukokinin (LK) leads to a high percentage of arrhythmic flies (65.4\%). LK is expressed in only four non-clock neurons in the adult brain: two in the lateral horn (LHLK neurons) and two in the SEZ. Because of their location and their projections to the dorsal region of the brain, they focused on the LHLK neurons and measured their activity in constant conditions using GCaMP6. Activity of these neurons peaks at the end of the subjective day and reaches a minimum at the end of the subjective night. Interestingly, the LHLK neurons are indirectly inhibited by PDF, suggesting that $\mathrm{PDFR}^{+}$neurons modulate the activity of these neurons (Cavey et al., 2016). However, we do not know whether the DN1p are directly connected with the LHLK neurons (Figure 3C). Finally, the authors confirmed the rhythmic activity of this circuit by recording the neuronal activity from neurons that express the 
leukokinin receptor and project to the proximal region of the LHLK neurons (LHLKR neurons). Interestingly, activation of LHLKR neurons using the R65C07 driver line, for 1 day in DD, changes the phase of locomotor activity. When these neurons are active, flies are constantly active during the subjective day but decrease their activity during the entire subjective night instead of showing a wild-type peak of activity at the end of the subjective day (Cavey et al., 2016). Hence, although the pattern of activity is different from controls and the phase is not restricted to the subjective evening, they are still rhythmic, suggesting that the LHLK circuit is downstream of the DN1p to phase locomotor behavior in DD. However, it is important to consider that the R65C07 driver is expressed in many other cells in the brain, and hence the behavior observed may not be driven by the LHLKR neurons.

In summary, in DD and constant temperature, the LN-MO relays the rhythmicity, while the DN1p shape and phase the locomotor activity toward the subjective evening (Figure 3A) but the downstream circuits remain ambiguous. How can we explain the specific implication of these two groups of neurons in the control of the locomotor rhythms in DD? There are nonclock cells that express PDFR (Im and Taghert, 2010), such as Ring neurons ( $\mathrm{R}$ neurons) in the ellipsoid body (EB), a structure that belongs to the central complex, the integration center for locomotion (Young and Armstrong, 2010). Hence, the activity of some of these R neurons could be directly influenced by diffusible PDF and therefore their rhythm could be under direct control of the PDF neurons (Liang et al., 2019). While the sLNv pacemaker would control the pace of these $\mathrm{R}$ neurons via PDF, the DN1p could phase the same or other neurons that would belong to the central complex. Although the DN1p do not physically interact with $\mathrm{R}$ neurons in the $\mathrm{EB}$, indirect connections exist (Guo et al., 2018; Lamaze et al., 2018a) (see final chapter: "Role of DN1p in Temperature-Dependent Sleep Regulation”).

\section{THE ROLE OF DN1p IN REGULATING LOCOMOTOR ACTIVITY IN LIGHT-DARK CYCLES AND CONSTANT MILD TEMPERATURES}

Interestingly, light intensity and temperature modulate the evening output of the DN1p (Figure 1C) (Zhang Y. et al., 2010). At $25^{\circ} \mathrm{C}$ the DN1p-driven evening anticipation is only visible at low light intensity ( $\leq 50 \mathrm{Lux})$. However, when the temperature drops to $20^{\circ} \mathrm{C}$ we can observe this output also under higher light intensities (Zhang Y. et al., 2010). How does the environment influence these DN1p outputs?

While a clock in the PDF cells is not necessary for morning anticipation (Stoleru et al., 2004; Zhang Y. et al., 2010), PDF neuropeptide or the presence of the PDF cells are (Renn et al., 1999). Interestingly, rescuing $p d f r$ only in the DN1p restores morning anticipation (Zhang L. et al., 2010). Clock proteins in the DN1p cycle with high amplitude in LD, even in the absence of either Pdf or cry (Cusumano et al., 2009). However, the fact that $P d f$ mutant flies do not show a morning anticipation suggests that the neuronal activity of the DN1p, and therefore their output, depends on the neuropeptide PDF. Indeed, a shot of PDF excites the DN1p in a PKA independent manner (Seluzicki et al., 2014). This suggests that in addition of maintaining a robust molecular rhythm, PDF promotes the morning anticipation output via modulating DN1p neuronal activity (Figure 2). The rhythmic neuronal activity of DN1p depends on the sodium leak channel narrow abdomen (na) (Nash et al., 2002; Flourakis et al., 2015). Remarkably, na $a^{\text {har }}$ mutants lose their morning anticipation and also, the startle response to light-on (Nash et al., 2002; Zhang L. et al., 2010). Both behaviors are restored when na expression is rescued in the DN1p (Zhang L. et al., 2010). In $g l^{60 j}$ mutants, which lack all retinal photoreceptors and the DN1p, both morning anticipation and startle response are absent as well (Helfrich-Förster et al., 2001). This suggests that DN1p are necessary for the morning peak, but they need PDF at the end of the night to induce the increase of locomotion around dawn.

The second DN1p output regulates the evening anticipation. However, this output is highly sensitive to the surrounding environment, as for example, higher light intensities inhibit it (see above and Figure 1C). Interestingly, the evening peak does become visible at higher light intensity in the absence of PDF or when its expression is reduced by half $\left(p d f^{0} /+\right)$ (Chatterjee et al., 2018). Furthermore, the PDF level is influenced by light intensity, as its expression increases with light (Chatterjee et al., 2018). From a yeast one-hybrid screen for transcriptional regulators of $P d f$ the nuclear receptor $H r 38$ was found as a potential candidate (Mezan et al., 2016). Knock down of Hr38 in PDF neurons leads to a decrease of PDF levels (Mezan et al., 2016) and consequently, the DN1p evening output becomes visible under high light condition (Chatterjee et al., 2018).

Intriguingly, this opposite effect of the PDF on DN1p outputs is comparable to the temperature effect on the morning and evening peaks (Majercak et al., 1999). Historically, the first $p d f r$ mutant described was named han, which means "cold" in Korean (Hyun et al., 2005). However, apart from this interesting coincidence, it is not known if PDF levels vary with temperature.

The DN1p show heterogeneity in their response to PDF. Some respond positively to PDF neuron activation, others are inhibited (Chatterjee et al., 2018). This could explain the antagonistic effect of PDF on DN1p outputs. The morning DN1p would be activated by PDF, while the evening DN1p would be inhibited (Chatterjee et al., 2018). How this antagonistic response is regulated is not known.

One possibility is that PDF reception differs between morning and evening DN1p. The Pdfr locus encodes four isoforms with different coding exons, notably, isoforms $\mathrm{C}$ and $\mathrm{D}$ have an extra C-terminal coding exon in the intracellular part of the receptor (FlyBase), suggesting distinctive functions downstream of PDF reception. The Pdfr mutant allele $h a n^{5304}$ causes a deletion of all seven transmembrane domains (Hyun et al., 2005). Hence, it is unlikely that the mutated protein will localize at the cell surface. han $^{5304}$ mutants show a behavioral phenotype equivalent to Pdf null flies: absence of morning anticipation, an advance of the evening peak in LD and arrhythmic behavior in DD. However, the mutant allele $h a n^{3369}$, which leads to a partial deletion of the $\mathrm{C}$ terminal domain potentially affecting the $\mathrm{C}$ and $\mathrm{D}$ isoforms only, maintains its morning anticipation, while causing arrhythmicity 
in DD (Hyun et al., 2005; Im and Taghert, 2010). This suggests that PDF may differentially affect neurons depending on the specific $P d f r$ isoforms they express. However, the $\mathrm{CRY}^{+}$vGlut $^{+}$ DN1p are sufficient to drive morning anticipation in standard LD (Chatterjee et al., 2018) and PDFR-MYC, a construct faithfully reporting PDFR expression, is exclusively expressed in the CRY ${ }^{+}$ DN1p (Im and Taghert, 2010). Therefore, we propose that PDF promotes the activity of the $\mathrm{CRY}^{+}$morning DN1p neurons, which in turn inhibit the activity of the evening DN1p.

\section{ROLE OF DN1p IN TEMPERATURE ENTRAINMENT}

The brain clock can be entrained by light and temperature (Wheeler et al., 1993). While about half of the clock neurons in the brain expresses cry and therefore can be entrained by light in the absence of a functional visual system (Emery et al., 2000), they cannot be entrained by temperature in the absence of the periphery, and more specifically, the chordotonal organs and aristae (Sehadova et al., 2009; Yadlapalli et al., 2018). In nocte mutants, the chordotonal organs are structurally defective, and flies do not properly entrain to TC, while synchronization to light is normal (Glaser and Stanewsky, 2005; Sehadova et al., 2009; Chen et al., 2018). Wild-type flies can be entrained to TC even with a small temperature variation of only $2^{\circ} \mathrm{C}$ (Chen et al., 2015; Yoshii et al., 2005). The Drosophila ionotropic receptor IR25a is specifically required for synchronization to such lowamplitude TC $\left(2^{\circ} \mathrm{C}\right)$ in LL or DD (Chen et al., 2015). Since IR25a mutants do not affect high-amplitude temperature entrainment, we can therefore propose that synchronization to low- and high-amplitude TC uses different molecular and potentially neuronal thermo-circuits. Interestingly, TIM oscillations respond differently to the absence of this ionotropic receptor depending on the light condition. In low amplitude LLTC, TIM peaks between ZT16 and ZT18 in all wild-type clock neurons analyzed in this study. In IR25a mutants, however, TIM is constantly low in the DN1p and DN2 and its oscillations are strongly disturbed in the PDF neurons, but are not, or only weakly affected in the LNd and DN3 (Chen et al., 2015), suggesting an IR25a independent mechanism for their entrainment to low amplitude TC in LL. In the same low amplitude TC during DD, TIM peaks at different phases in the clock neurons: At ZT16 in the LN, at ZT10 in the DN2, and between ZT10 and ZT16 in the DN1p and DN3. In IR $25 a$ mutants, TIM is at constitutively low levels in the LN, but oscillations remain unchanged in the DN2, DN3 and in the DN1p, although the amplitude is dampened and the peak narrowed. The DDTC situation is difficult to interpret because one could think that if clock neurons become insensitive to temperature entrainment they should maintain their oscillations and free run. Hence, we do not understand why TIM levels are low and flat in the LNd and sLNv. Nonetheless, in low-amplitude LLTC, the effect of IR25a on TIM expression in the DN1p and DN2 is very clear. Furthermore, when DN1p or DN2 neuronal activity is inhibited using tetanus toxin, flies fail to synchronize to a low amplitude TC in LL (Chen et al., 2015), also supporting a role for the DN1p (and DN2) in these conditions.
In the wild, the daily variation of temperature follows the sun. Hence, to test whether we can distinguish a light oscillator from a temperature one, like in plants (Michael et al., 2003), we need to uncouple light and TC. Using an environmental uncoupling protocol, where LD and TC were offset by $6 \mathrm{~h}$, TIM expression in the lateral neurons followed the LD regime, while the dorsal neurons were preferentially entrained by temperature (Miyasako et al., 2007). Later, applying a protocol with exactly opposite $\mathrm{LD}$ and TC, only the $\mathrm{CRY}^{-} \mathrm{DN} 1 \mathrm{p}$, DN2, and DN3 followed the TC (Yoshii et al., 2010). Using a different sensory conflict protocol and PER immunostaining (which is less sensitive to light inputs), Harper et al. (2016) observed that only the DN2 and DN3 follow the TC. However, in the absence of cry, PER oscillations in all clock neurons analyzed followed the TC, contrary to what was observed during opposite LD and TC, where the LN-MO maintained its phase in accordance with the LD cycle (Yoshii et al., 2010). Nonetheless, it is striking to observe that in $c r y^{0}$ flies, PER oscillates with the highest amplitude in DN1p and DN2, whether light and temperature oscillations are in phase or not (Harper et al., 2016).

The TRPA channel pyrexia ( $p y x)$ is specifically required for synchronization to cold TC $\left(20-16^{\circ} \mathrm{C}\right)$ (Wolfgang et al., 2013). While IR25a is expressed in the chordotonal neurons (Chen et al., 2015), pyx is expressed in the cap cells of the chordotonal organs as well as in the peripheral nervous system (PNS) (Wolfgang et al., 2013; Roessingh et al., 2019). pyx ${ }^{3}$ mutants do not properly synchronize to cold TC in DD and free run instead (Roessingh et al., 2019). Interestingly, while PER oscillations in the sLNv of $p y x^{3}$ mutants seem to free run during the shifted TC, they are dampened in the DN1p.

Although pyx and IR25a are both expressed in chordotonal organs, they play a role in specific TC conditions, and differently affect temperature synchronization of various clock neurons. Chordotonal organs are present all over the Drosophila body. However, it is unclear how the temperature information is transferred from the chordotonal organs to the clock neurons. The light conditions are also an important factor because the DN1p are differentially affected by the absence of IR25a in presence or absence of light.

In summary, the DN1p are sensitive to temperature entrainment when they do not receive oscillating light inputs. Their molecular synchronization is strongly affected when chordotonal function or structure are disturbed (Chen et al., 2015, 2018; Roessingh et al., 2019). In low amplitude TC, their neuronal activity is required for behavioral entrainment. However, about half of them express cry and in a temperaturelight conflict paradigm, they are synchronized with the light (Harper et al., 2016).

\section{ROLE OF THE DN1P IN TEMPERATURE-DEPENDENT SLEEP REGULATION}

Sleep is a fundamental physiological process regulated by both homeostatic and circadian mechanisms. Sleep need accumulates 
during wakefulness and is released during sleep. In DD, the clock phases sleep (Shaw et al., 2000). However, a nocturnal mouse can switch to diurnality when its environment changes (food access and temperature) suggesting that the environment, independent of its entrainment function, can phase sleep (van der Vinne et al., 2014).

All animals with a complex central nervous system sleep, though the sleep pattern differs between species (Cirelli and Tononi, 2008). D. melanogaster is considered crepuscular for chrono biologists but diurnal for sleep biologists. In standard LD conditions, flies are mostly active around light transitions, explaining the crepuscular classification. In between, they show sleep-like behaviors, and because night sleep lasts longer than day sleep (also called siesta), they have been classified as diurnal. It is today clear that day and night sleep are differently regulated in flies (Ishimoto et al., 2012) and of different quality (Van Alphen et al., 2013). Consequently, siesta and night sleep might use different neuronal circuits.

In standard LD conditions, mutants for the peptide DH31 sleep slightly more than controls (Kunst et al., 2014). Especially during the morning anticipation period at the end of the night, flies are sleepier in the absence of DH31 (Kunst et al., 2014). Interestingly, some of the DN1p express this neuropeptide, and their activation delays day sleep onset and decreases sleep at the second half of the night (Kunst et al., 2014). This suggests that the $\mathrm{DH} 31^{+} \mathrm{DN} 1 \mathrm{p}$ play a role in wakefulness around the light-on transition. Since this behavioral phenotype was obtained using ectopic DN1p activation, the question is if, and under which conditions, these clock neurons promote arousal in the morning?

An increase of temperature above $30^{\circ} \mathrm{C}$ phase-shifts the siesta, and both its onset and offset are delayed. These high temperatures also advance the offset of night sleep (Lamaze et al., 2017; Figures $\mathbf{4 A}, \mathbf{B}$ ). The delay of the siesta offset and the advance of the night sleep offset mirror the evening and morning anticipatory activity behaviors at warm temperature $\left(\geq 27^{\circ} \mathrm{C}\right)$ (Majercak et al., 1999; Figure 4C). Interestingly, the delay of the siesta onset is also clock-dependent (Lamaze et al., 2018a). Hence, both the onset and the offset of the siesta phase-shift in a clock dependent manner (Figure 4B). Interestingly, inhibition of the DN1p neuronal activity at warm temperature $\left(\geq 30^{\circ} \mathrm{C}\right)$, using shibire ${ }^{t s}\left(s h i^{t s}\right)$ (Dubnau et al., 2001), inhibits the delay of the siesta onset but not its offset (Lamaze et al., 2017; Figure 6A). This suggests that the DN1p promote morning arousal at warm temperature but their neuronal activity at the end of the day is not responsible for the phase delay of the siesta offset. Consistent with this, several groups have shown that the neuronal activity of the DN1p cycles along the day with a peak in the early morning (Flourakis et al., 2015; Liang et al., 2016) and a trough in the afternoon. Furthermore, stopping the clock in the DN1p via expressing a dominant negative form of $c y c$ (Tanoue et al., 2004) does not affect the normal activity and sleep pattern at $22^{\circ} \mathrm{C}$ (Figure 5A), but their behavior becomes aberrant at $31^{\circ} \mathrm{C}$ (Figure 5B). Notably, during the day, the siesta is not restricted to a defined time of day. Interestingly, the night sleep is completely reversed compared to controls and the flies sleep when they should be awake and vice versa (Figure 5B). Therefore, the DN1p are essential for phasing behavior at temperatures $\geq 30^{\circ} \mathrm{C}$, but at mild temperature, they do not play a fundamental role in controlling the locomotor pattern.

In contrast, Guo et al. (2016) have proposed that the DN1p promote sleep at mild temperatures, suggesting to have identified the first sleep-promoting clock neurons in Drosophila. They notably proposed that the DN1p promote sleep in the late day via inhibiting the LN-EO through a glutamatergic pathway (Guo et al., 2016). However, the LNEO is sufficient to promote the increase of locomotion in the early evening before the light-off transition (Grima et al., 2004). Furthermore, live calcium imaging revealed that in standard LD the neuronal activity of the LNd oscillates in antiphase with the DN1p (Liang et al., 2016). Therefore, it is difficult to comprehend the relevance of an inhibitory action from DN1p sleep-promoting neurons on the LN-EO at a time when the DN1p are less active than the LN-EO. However, the locomotor behavior pattern changes with temperature, notably, at warm temperature $\left(\geq 29^{\circ} \mathrm{C}\right)$ the evening anticipation is delayed compared to standard mild temperature (Majercak et al., 1999). Can the sleep-promoting DN1p inhibit the LNd in this condition and therefore delay the late-day wakefulness? This seems unlikely since the inhibition of the DN1p at $\geq 30^{\circ} \mathrm{C}$ does not inhibit the delay of the siesta offset (Lamaze et al., 2017; Figure 6A), suggesting that they are already inhibited at this time of day and temperature level. Finally, cooler temperatures $\left(<20^{\circ} \mathrm{C}\right)$ advance the evening activity (Majercak et al., 1999), and the DN1p evening locomotor output is also promoted at cooler temperature $\left(20^{\circ} \mathrm{C}\right)$ (Zhang Y. et al., 2010). Interestingly, Yadlapalli et al. (2018) have observed that cooling in the afternoon activated DN1p neuronal activity, suggesting again that the DN1p are pro-arousal. It would be interesting to test the role of a potential cooperation between LN-EO and DN1p to control the phase of the evening peak at cooler temperatures.

The thermoreceptor $\mathrm{dTrpA} 1$ phases the siesta and the morning anticipation at warm temperatures $\left(>29^{\circ} \mathrm{C}\right)$ (Roessingh et al., 2015; Lamaze et al., 2017). Two groups of dTrpA1expressing neurons, the $p p k$ and the $d \operatorname{Tr} p A 1[\mathrm{SH}]$ neurons, project to the dorso-posterior area of the brain where the DN1p, the LN-EO, and also the sLNv (LN-MO) project (Figure 6C). Downregulation of dTrpA1 expression in either the ppk neurons or the dTrpAl[SH] neurons, strongly dampened the phase shift of the siesta (both onset and offset) and the phase advance of the morning anticipation normally observed at warm temperature $\left(\geq 30^{\circ} \mathrm{C}\right)$ (Lamaze et al., 2017). Since an inhibition of DN1p neuronal activity does not affect the delay of the siesta offset, we can propose that the dTrpA1expressing neurons projecting to the dorso-posterior area of the brain, phase the siesta offset and onset in response to warm temperatures by directly interacting with the LN-EO and the DN1p, respectively.

The DN1p are a heterogeneous group, both transcriptomically and anatomically. Two sub-populations can be distinguished: 


\section{A}

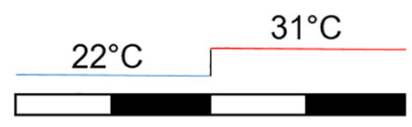

B
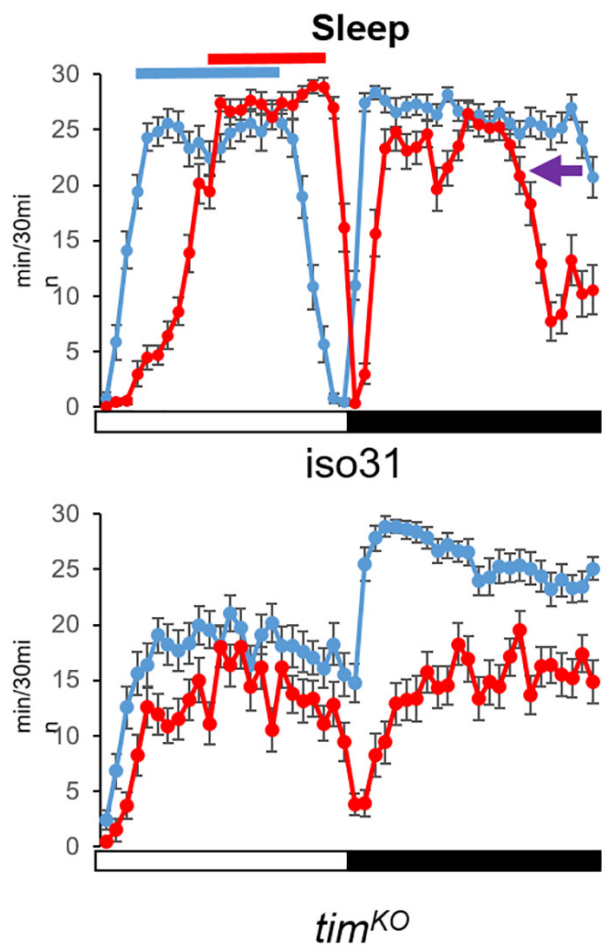

C
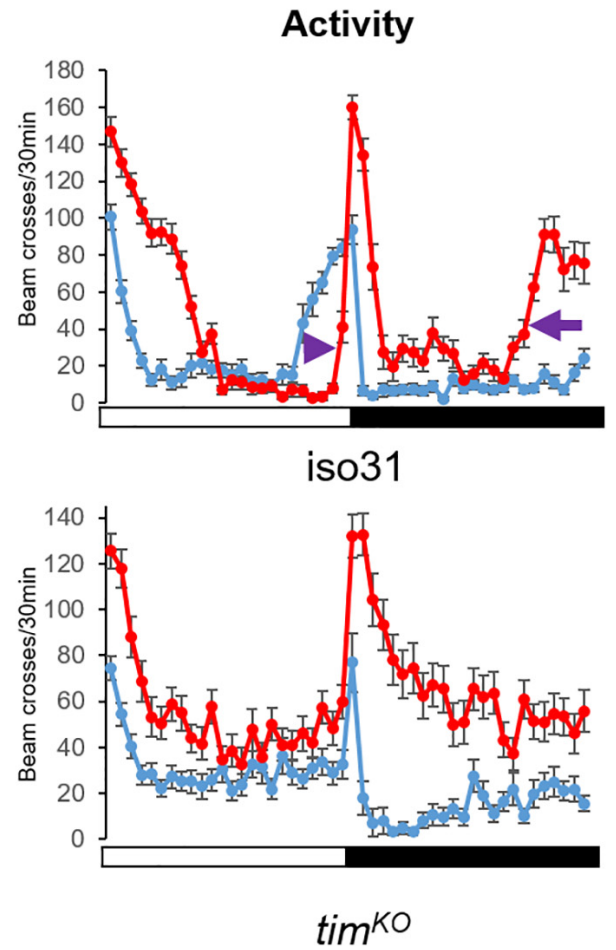

FIGURE 4 | Warm temperature differentially affects day and night sleep. (A) Temperature protocol: White bar $12 \mathrm{~h}$ of light, dark bar $12 \mathrm{~h}$ of darkness. Flies were kept at $22^{\circ} \mathrm{C}$ the first day (blue line). The next day, temperature increased at light-on to $31^{\circ} \mathrm{C}$ for $24 \mathrm{~h}$ (red line). (B) Average sleep trace of control flies (iso31, $\mathrm{N}=31$ ) and clock mutant males [tim ${ }^{K O}$ (Lamaze et al., 2017), $N=32$ ] (bottom graph). Sleep was measured as the sum of 5 min of inactivity per half hour. At warm temperature, wild-type flies delay their siesta. Both onset and offset are delayed (the red line shifts to the right compared to the blue line). At night and $31^{\circ} \mathrm{C}$, flies sleep less. Notably, sleep offset is advanced compared to $22^{\circ} \mathrm{C}$ (purple arrow). On the other hand, the siesta of clock mutant flies is less affected, i.e., we do not observe a robust phase shift. Night sleep however is strongly dampened at $31^{\circ} \mathrm{C}$. (C) Average locomotor activity of the same flies as in Graph (B). The activity is measured as the sum of beam-crossings per half hour. We can observe the delay of the evening anticipation in iso31 flies (purple arrowhead), which corresponds to the delay of sleep offset. And while the morning anticipation is barely visible at $22^{\circ} \mathrm{C}$ (but clearer when looking at the sleep profile), at $31^{\circ} \mathrm{C}$ we can observe a clear advanced morning anticipation (purple arrow). Although clock mutant flies increase their locomotion in response to warm (startle response to light-on switches from 80 beam crosses $/ 30$ min at $22^{\circ} \mathrm{C}$ to 130 at $31^{\circ} \mathrm{C}$ ), they do not present a phase shift of the trough of activity as observed in wild-type flies. At night, clock mutants maintain a high level of activity. Hence, we can see that the delay of the siesta at warm temperatures is clock dependent and independent of the acute response to temperature increase. Error bars represent standard error of the mean.

one projecting anteriorly and terminating in the central domain of the small unit of the anterior optic tubercle (sAOTUc), and which are therefore called aDN1p (Figure 6C). The other group projects ventrally and posteriorly, and is therefore called vDN1p (Lamaze et al., 2018a). Guo et al. (2017) have described a driver line (JRC_SS00781 or Spl-DN1p) that restricts the number of sleep-promoting DN1p and their neurons belong exclusively to the vDN1p group (Lamaze et al., 2018a and Supplementary Figure 1 of Guo et al., 2017). Surprisingly, using an independent combination of driver lines to activate $\mathrm{CRY}^{-}$DN1p, which do not project to the AOTU, the same lab found no sleep promotion (Guo et al., 2018), questioning the identity of the sleep promoting neurons.

The AOTU is a neuropil receiving visual input from specific neurons in the medulla (Omoto et al., 2017; Timaeus et al., 2017).
At $31^{\circ} \mathrm{C}$, activation of tubulo-bulbar neurons $\left(\mathrm{TuBu}_{c}\right)$ that project dendrites to the sAOTUc inhibits the delay of the siesta onset but not the delay of the siesta offset, suggesting a sleep-promoting function. Importantly, DN1p inhibition at warm temperature $\left(\geq 30^{\circ} \mathrm{C}\right)$ in the morning promotes activity of $\mathrm{TuBu}_{c}$ neurons, while activation of the DN1p in the warm afternoon inhibits the TuBu neuronal activity (Lamaze et al., 2018a). This suggests that the aDN1p promote arousal in the morning via the inhibition of their direct downstream target, the $\mathrm{TuBu}_{c}$ neurons. The $\mathrm{TuBu}$ neurons project to a microglomeruli structure called the lateral triangle (or bulb), where they interact with $\mathrm{R}$ neurons, which in turn project axons forming rings in the EB (Omoto et al., 2017; Timaeus et al., 2017). The sleep-promoting $\mathrm{TuBu}_{c}$ neurons interact with a subset of R2 neurons in the superior region of the 
A
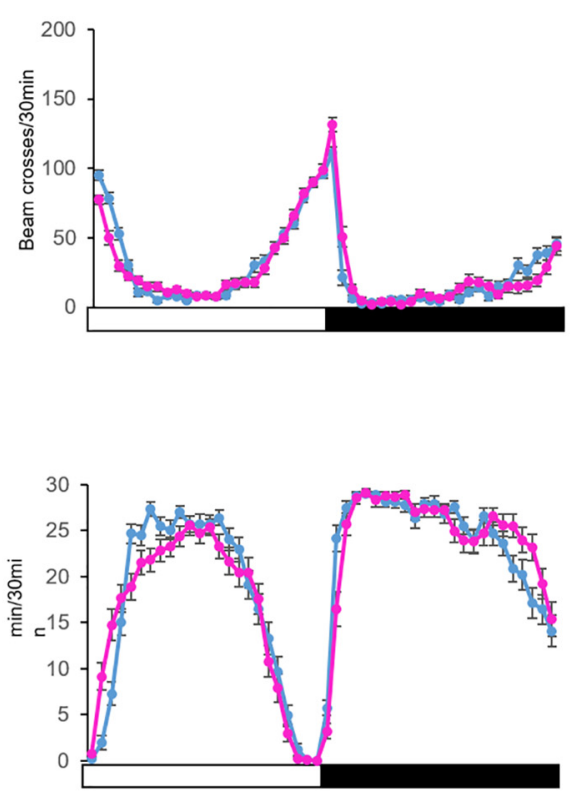

B
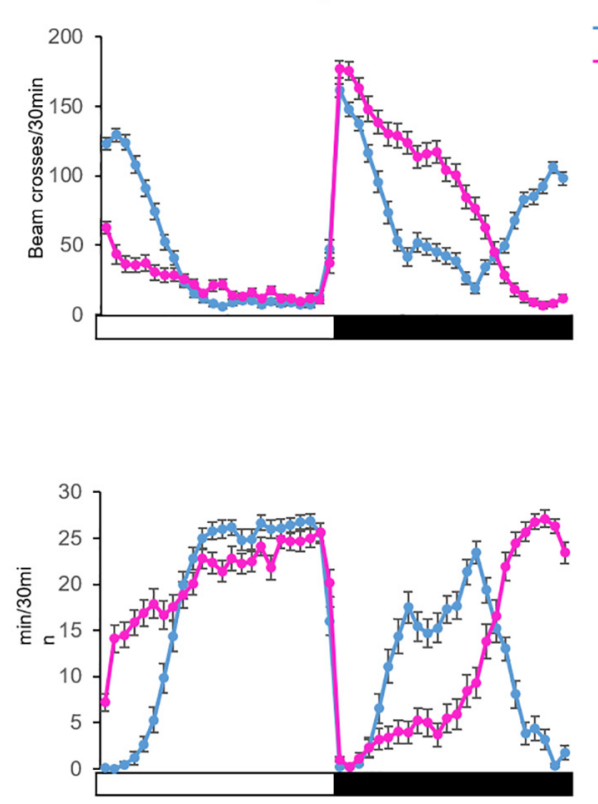

FIGURE 5 | The DN1p clock is necessary to adapt locomotor behavior during warm temperatures. (A) Top: the locomotor activity monitored at $22^{\circ} \mathrm{C}$ on day 1 ; Bottom: the sleep trace in the same condition from the same flies. (B) Activity (Top) and sleep (Bottom) pattern of the same flies monitored the next day at $31^{\circ} \mathrm{C}$. We

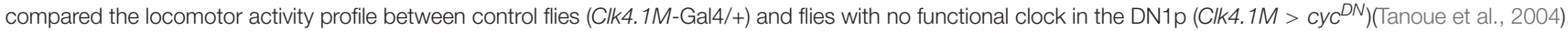
$N(C / k 4.1 M-G a l 4 /+)=51 ; N(C / k 4.1 M>c y C D N)=48$; error bars represent standard error of the mean.

A

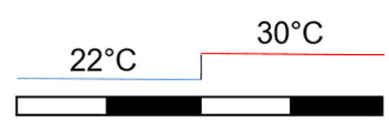

DN1p $>$ shits

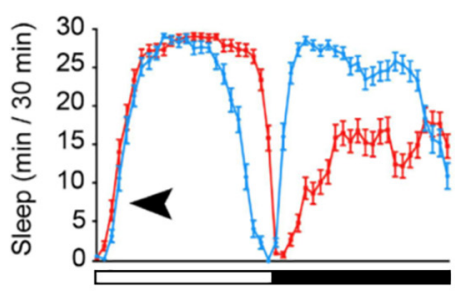

B

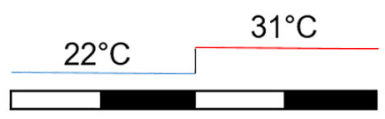

$\mathrm{R} 2>\operatorname{TrpA} 1$
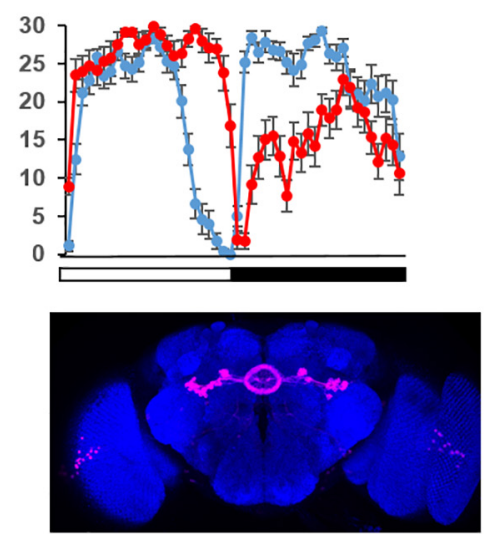

C

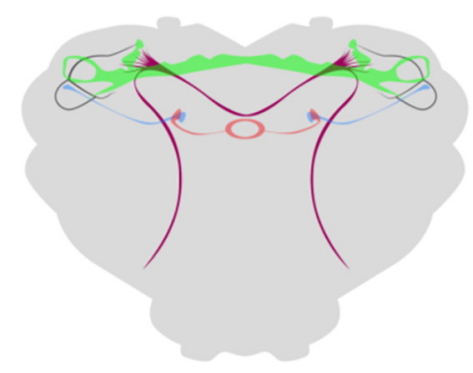

- TrpA1-expressing neurons

- aDN1p

$-\mathrm{TuBu}_{\mathrm{c}}$

- R2 neurons

FIGURE 6 | Timing the siesta onset during warm temperatures. (A) Average sleep trace of male flies expressing shits in the DN1p. The temperature protocol is sketched above the graph. The sleep trace was copied from Lamaze et al. (2017) the black arrowhead indicates the absence of delay of the siesta onset at $30^{\circ} \mathrm{C}$. (B) Average sleep trace of male flies expressing TrpA1 in R2 neurons, using the same driver as in Lamaze et al. (2018a) (R20D01-Gal4). The brain picture of the expression pattern of R20D01 is a Z stack from the Janelia FlyLight database. TrpA1 is inactive at $22^{\circ} \mathrm{C}$ but active at $31^{\circ} \mathrm{C}$. Note the remarkable symmetry between flies with inactivated DN1p and activated R2 neurons. $N(R 2>\operatorname{TrpA1})=20$. (C) Sketch of the neuronal circuit involved in timing the siesta based from Lamaze et al. (2017, 2018a,b). The dorsal-projecting TrpA ${ }^{+}$neurons play an essential role in phasing the siesta with warm temperatures (Lamaze et al., 2017). They physically interact with DN1p clock neurons (Lamaze et al., 2017). The aDN1p directly inhibit a subset of TuBuc neurons in the central domain of sAOTU (Lamaze et al., 2018a). These $\mathrm{TuBu}_{c}$ neurons promote sleep when activated at warm temperature. They interact in the superior region of the bulb with a subset of R2 neurons (Lamaze et al., 2018a). 
bulb (Lamaze et al., 2018a; Figure 6C). Importantly, activation of these neurons at $31^{\circ} \mathrm{C}$ inhibits the delay of the siesta onset (Figure 6B), suggesting that $\mathrm{TuBu}_{c}$ neurons activate $\mathrm{R} 2$ neurons to promote sleep in the morning. Recently, Liang et al. (2019) measured rhythmic neuronal activity in different groups of $\mathrm{R}$ neurons including $\mathrm{R} 2$ (also called R4m). The $\mathrm{EB}$ is a neuropil structure integrating visual inputs and plays an important role in navigation (Sun et al., 2017). Hence, in order to fall asleep during daytime, this structure needs to decrease its sensitivity to visual inputs in order to increase the threshold of response. We can therefore propose that a subset of $\mathrm{TuBu}_{c}$ neurons that receive time information input from the DN1p promote sleep by decreasing the sensitivity of the $\mathrm{EB}$ to visual input via the activation of a subset of R2 neurons. During the night, flies do not receive any light input and activation of these $\mathrm{R}$ neurons does not inhibit the sleep loss induced by temperature (Figure 6B). D. melanogaster is crepuscular. Hence, they are more active at dawn, especially at warm temperature. A subset of DN1p promote arousal during that time, according to the ambient temperature level.

\section{CONCLUSION AND FUTURE DIRECTIONS}

The brain clock controls sleep/wake rhythms. In D. melanogaster, only 150 neurons express clock genes and yet, we do not know how their time information is integrated by the central nervous system. The DN1p are a very peculiar group. First, their development correlates with the development of the visual system. glass encodes a transcription factor required for photoreceptor development (Moses et al., 1989). $g l^{60 j}$ mutants have a profound defect of the visual system development and in addition, the DN1p fail to differentiate. This suggests a developmental and/or functional relationship between these clock neurons and the visual system (Helfrich-Förster et al., 2001; Klarsfeld et al., 2004). Second, although they express clock genes, the DN1p are not autonomous, and their DD rhythm entirely depends on the sLNv pacemaker neurons and the neuropeptide PDF (Klarsfeld et al., 2004). Finally, although the cells within this group are synchronized to the same phase (molecular clock but also presumably neuronal activity), the DN1p can drive both morning and evening activity under specific environmental conditions (Zhang Y. et al., 2010). This suggests a further separation within this group of at least two clusters.

The DN1p's evening activity is inhibited by strong light $(\geq 500$ Lux) and is only visible at $25^{\circ} \mathrm{C}$ under low light condition $(\leq 50$ Lux). Interestingly, in DD the main peak of locomotor activity happens during the subjective evening. This phase is under the control of the DN1p (Chatterjee et al., 2018), suggesting that the DN1p that drive the evening activity under LD low light condition are the same as the ones phasing the locomotor activity in DD. Can we define the two clusters of DN1p more precisely? CRY is required to phase the onset of the siesta at warm temperatures (Lamaze et al., 2017) and the CRY- DN1p do not project to the AOTU (Chatterjee et al., 2018; Guo et al., 2018). Hence, we can propose that the aDN1p, which promote wakefulness in the warm dawn, are $\mathrm{CRY}^{+}$. Furthermore, a clock in the vGlut ${ }^{+}$DN1p is sufficient to drive the morning anticipation (Chatterjee et al., 2018), suggesting that the aDN1p are $\mathrm{CRY}^{+}$and vGlut ${ }^{+}$. However, two of the CRY ${ }^{+}$neurons are vGlut $^{-}$(Chatterjee et al., 2018), indicating that the DN1p-driven evening activity is under the control of a mix of $\mathrm{CRY}^{+}$and $\mathrm{CRY}^{-}$ vGlut $^{-}$neurons.

The distinction of DN1p clusters is less clear when we focus on the projection to the PI. The DN1p physically interact with different neuroendocrine cells in the PI. Since the DH44 neurons do not send axonal projections to the thoracic ganglion, or to the central complex, and because downregulation of its receptor DH44R1 does not lead to arrhythmicity (King et al., 2017), it seems unlikely that these neuropeptidergic neurons influence locomotor rhythms in DD. However, this circuit could play a role in feeding rhythm and/or rhythmic metabolic processes (Dus et al., 2015; Figure 3B). The DH44 neurons are not the only neuroendocrine cells interacting with the DN1p (Barber et al., 2016; Cavey et al., 2016), and the DN1p are not the only clock neurons projecting to this region (Schubert et al., 2018). Hence, it would be of high interest to investigate the role of the clock neurons in the control of the PI-corpora cardiaca/corpora allata activity. This circuit is considered to be the functional homolog of the vertebrate hypothalamus-hypophysis circuit (Buch and Pankratz, 2009).

The central complex in Drosophila could be compared to the processor of a computer. It is the part of the brain where the computation happens in order to drive the appropriate behavior in response to the various inputs the brain receives. The central complex is composed of several neuropil structures, including the fan shaped body and the EB. A direct connection between the DN1p and neurons of the central complex is not known. However, the DN1p interact with cells that do connect to neurons projecting to the central complex. The aDN1p interact with a subset of $\mathrm{TuBu}_{c}$ neurons which in return contact a subset of $\mathrm{R} 2$ neurons in the superior part of the bulb. The symmetry of the behavior between flies with silenced DN1p and activated R2 neurons is striking (Lamaze et al., 2017; Figure 6B). Recently, Liang et al. (2019) have measured the neuronal activity of different groups of $\mathrm{R}$ neurons along $24 \mathrm{~h}$ during $\mathrm{LD}$ and the first day of DD. Interestingly, the group of $\mathrm{R}$ neurons that show the most homogeneous rhythmic activity are the R5 [confusingly called R2 in this study (Liang et al., 2019)]. We do not know however, whether activation or inhibition of R5 neurons affect the locomotor activity in DD, notably whether their inhibition would change the phase of the locomotor behavior in DD. Nonetheless, it is interesting to note that many neuronal groups in the brain display rhythmic activity (Barber et al., 2016; Cavey et al., 2016; Liang et al., 2019), and most of them do not interact directly with clock neurons. Actually, apart from the $\mathrm{TuBu}_{c}$ neurons (Lamaze et al., 2018a) and the PI neurons (Cavanaugh et al., 2014; Barber et al., 2016) which interact 
with the DN1p, none of the other groups directly interact with the DN1p. However, it is most likely that rhythmic signals end up in the central complex to control locomotor patterns. Interestingly, PDFR is expressed in different $\mathrm{R}$ neurons, including $\mathrm{R} 2$ and internal rings (Im and Taghert, 2010; Liang et al., 2019). Hence, it is totally conceivable that PDF neurons control the DD rhythm via secreted PDF action on the central complex, while the DN1p control the phase of the locomotor activity pattern via their indirect interaction with the EB.

To conclude, although the DN1p cannot be considered as a circadian oscillator, they play an essential role in phasing clockcontrolled behaviors. Since the phase of a circadian behavior is the result of an integration of the environmental status with the time of day, this group of clock neurons seems to function at the crossroad between environmental input, in particular temperature, and the internal clock.

\section{REFERENCES}

Barber, A. F., Erion, R., Holmes, T. C., and Sehgal, A. (2016). Circadian and feeding cues integrate to drive rhythms of physiology in Drosophila insulin-producing cells. Genes Dev. 30, 2596-2606. doi: 10.1101/gad.288258.116

Benito, J., Houl, J. H., Roman, G. W., and Hardin, P. E. (2008). Hardin. the blue-light photoreceptor cryptochrome is expressed in a subset of circadian oscillator neurons in the Drosophila CNS. J. Biol. Rhythms 23, 296-307. doi: $10.1177 / 0748730408318588$

Buch, S., and Pankratz, M. (2009). Making metabolic decisions in Drosophila. Fly 3, 74-77. doi: 10.4161/fly.3.1.7795

Cabrero, P., Radford, J. C., Broderick, K. E., Costes, L., Veenstra, J. A., and Spana, E. P. (2002). The Dh gene of Drosophila melanogaster encodes a diuretic peptide that acts through cyclic AMP. J. Exp. Biol. 205, 3799-3807.

Cavanaugh, D. J., Geratowski, J. D., Wooltorton, J. R., Spaethling, J. M., Hector, C. E., and Zheng, X. (2014). Identification of a circadian output circuit for rest: activity rhythms in Drosophila. Cell 157, 689-701. doi: 10.1016/j.cell.2014.02. 024

Cavey, M., Collins, B., Bertet, C., and Blau, J. (2016). Circadian rhythms in neuronal activity propagate through output circuits. Nat. Neurosci. 19, 587-595. doi: 10.1038/nn.4263

Chatterjee, A., Lamaze, A., De, J., Mena, W., Chélot, E., and Martin, B. (2018). Reconfiguration of a multi-oscillator network by light in the Drosophila circadian clock. Curr. Biol. 28, 2007-2017.e4. doi: 10.1016/j.cub.2018.04.064

Chen, C., Buhl, E., Xu, M., Croset, V., Rees, J. S., and Lilley, K. S. (2015). Drosophila ionotropic receptor 25 a mediates circadian clock resetting by temperature. Nature 527, 516-520. doi: 10.1038/nature16148

Chen, C., Xu, M., Anantaprakorn, Y., Rosing, M., and Stanewsky, R. (2018). Nocte is required for integrating light and temperature inputs in circadian clock neurons of Drosophila. Curr. Biol. 28, 1595-1605. doi: 10.1016/j.cub.2018.04. 001

Cirelli, C., and Tononi, G. (2008). Is sleep essential? PLoS Biol. 6:e216. doi: 10.1371/ journal.pbio.0060216

Cusumano, P., Klarsfeld, A., Chélot, E., Picot, M., Richier, B., and Rouyer, F. (2009). modulated visual inputs and cryptochrome define diurnal behavior in Drosophila. Nat. Neurosci. 12, 1431-1437. doi: 10.1038/nn.2429

Diaz, M. M., Schlichting, M., Abruzzi, K. C., Long, X., and Rosbash, M. (2019). Allatostatin-C/AstC-R2 is a novel pathway to modulate the circadian activity pattern in Drosophila. Curr. Biol. 29, 13-22. doi: 10.1016/j.cub.2018.11.005

Dubnau, J., Grady, L., Kitamoto, T., and Tully, T. (2001). Disruption of neurotransmission in Drosophila mushroom body blocks retrieval but not acquisition of memory. Nature 411, 476-480. doi: 10.1038/35078077

Dus, M., Lai, J. S.-Y., Gunapala, K. M., Min, S., Tayler, T. D., and Hergarden, A. C. (2015). Nutrient sensor in the brain directs the action of the brain-gut axis in Drosophila. Neuron 87, 139-151. doi: 10.1016/j.neuron.2015.05.032

\section{AUTHOR CONTRIBUTIONS}

AL wrote the manuscript and RS commented on it.

\section{FUNDING}

$\mathrm{AL}$ received funding from a WiRe (Women in Research, WWU) fellowship. AL and RS are supported by the DFG grant STA421/71.

\section{ACKNOWLEDGMENTS}

We thank Clara Lorber for helping with behavior experiments. We also thank Cédric Lamaze for the cartoon representing the downstream neuronal network of the aDN1p.

Emery, P., Stanewsky, R., Helfrich-Förster, C., Emery-Le, M., Hall, J. C., and Rosbash, M. (2000). Drosophila CRY is a deep brain circadian photoreceptor. Neuron 26, 493-504. doi: 10.1016/s0896-6273(00)81181-2

Flourakis, M., Kula-Eversole, E., Hutchison, A. L., Han, T. H., Aranda, K., and Moose, D. L. (2015). A conserved bicycle model for circadian clock control of membrane excitability. Cell 162, 836-848. doi: 10.1016/j.cell.2015. 07.036

Gentile, C., Sehadova, H., Simoni, A., Chen, C., and Stanewsky, R. (2013). Cryptochrome antagonizes synchronization of Drosophila's circadian clock to temperature cycles. Curr. Biol. 23, 185-195. doi: 10.1016/j.cub.2012.12.023

Glaser, F. T., and Stanewsky, R. (2005). Temperature synchronization of the Drosophila circadian clock. Curr. Biol. 15, 1352-1363. doi: 10.1016/j.cub.2005. 06.056

Grima, B., Chélot, E., Xia, R., and Rouyer, F. (2004). Morning and evening peaks of activity rely on different clock neurons of the Drosophila brain. Nature 431, 869-873. doi: 10.1038/nature02935

Guo, F., Chen, X., and Rosbash, M. (2017). Temporal calcium profiling of specific circadian neurons in freely moving flies. Proc. Natl. Acad. Sci. U.S.A. 114, E8780-E8787. doi: 10.1073/pnas.1706608114

Guo, F., Holla, M., Díaz, M. M., and Rosbash, M. (2018). A circadian output circuit controls sleep-wake arousal in Drosophila. Neuron 100, 624-635. doi: 10.1016/j.neuron.2018.09.002

Guo, F., Yu, J., Jung, H. J., Abruzzi, K. C., Luo, W., Griffith, L. C., et al. (2016). Circadian neuron feedback controls the Drosophila sleep-activity profile. Nature 536, 292-297. doi: 10.1038/nature19097

Hamasaka, Y., Rieger, D., Parmentier, M. L., Grau, Y., Helfrich-Förster, C., and Nässel, D. R. (2007). Glutamate and its metabotropic receptor in Drosophila clock neuron circuits. J. Comp. Neurol. 505, 32-45.

Harper, R. E., Dayan, P., Albert, J. T., and Stanewsky, R. (2016). Sensory conflict disrupts activity of the Drosophila circadian network. Cell Rep. 17, 1711-1718. doi: 10.1016/j.celrep.2016.10.029

Hector, C. E., Bretz, C. A., Zhao, Y., and Johnson, E. C. (2009). Functional differences between two CRF-related diuretic hormone receptors in Drosophila. J. Exp. Biol. 212, 3142-3147. doi: 10.1242/jeb.033175

Helfrich-Förster, C. (2019). Light input pathways to the circadian clock of insects with an emphasis on the fruit fly Drosophila melanogaster. J. Comp. Physiol. A Neuroethol. Sens. Neural. Behav. Physiol. doi: 10.1007/s00359-019-01379-5 [Epub ahead of print], .

Helfrich-Förster, C., Winter, C., Hofbauer, A., Hall, J. C., and Stanewsky, R. (2001). The circadian clock of fruit flies is blind after elimination of all known photoreceptors. Neuron 30, 249-261. doi: 10.1016/s0896-6273(01) 00277-x

Hyun, S., Lee, Y., Hong, S.-T., Bang, S., Paik, D., Kang, J., et al. (2005). Drosophila GPCR Han is a receptor for the circadian clock neuropeptide PDF. Neuron 48, 267-278. doi: 10.1016/j.neuron.2005.08.025 
Im, S. H., and Taghert, P. H. (2010). PDF receptor expression reveals direct interactions between circadian oscillators in Drosophila. J. Comp. Neurol. 518, 1925-1945. doi: 10.1002/cne.22311

Ishimoto, H., Lark, A. R., and Kitamoto, T. (2012). Factors that differentially affect daytime and nighttime sleep in Drosophila melanogaster. Front. Neurol. 3:24. doi: 10.3389/fneur.2012.00024

King, A. N., Barber, A. F., Smith, A. E., Dreyer, A. P., Sitaraman, D., and Nitabach, M. N. (2017). A peptidergic circuit links the circadian clock to locomotor activity. Curr. Biol. 27, 1915-1927.e5. doi: 10.1016/j.cub.2017.05.089

Klarsfeld, A., Malpel, S., Michard-Vanhée, C., Picot, M., Chélot, E., and Rouyer, F. (2004). Novel features of cryptochrome-mediated photoreception in the brain circadian clock of Drosophila. J. Neurosci. 24, 1468-1477. doi: 10.1523/ jneurosci.3661-03.2004

Konopka, R. J., and Benzer, S. (1971). Clock mutants of Drosophila melanogaster. Proc. Natl. Acad. Sci. U.S.A. 68, 2112-2116. doi: 10.1073/pnas.68.9.2112

Kunst, M., Hughes, M. E., Raccuglia, D., Felix, M., Li, M., Barnett, G., et al. (2014). Calcitonin gene-related peptide neurons mediate sleep-specific circadian output in Drosophila. Curr. Biol. 24, 2652-2664. doi: 10.1016/j.cub.2014.09.077

Lamaze, A., Krätschmer, P., Chen, K.-F., Lowe, S., and Jepson, J. E. (2018a). A Wake-Promoting circadian output circuit in Drosophila. Curr. Biol. 28, 3098-3105.e3. doi: 10.1016/j.cub.2018.07.024

Lamaze, A., Kratschmer, P., and Jepson, J. E. (2018b). A sleep-regulatory circuit integrating circadian, homeostatic and environmental information in Drosophila. bioRxiv [preprint]. doi: 10.1101/250829

Lamaze, A., Öztürk-Çolak, A., Fischer, R., Peschel, N., Koh, K., and Jepson, J. E. (2017). Regulation of sleep plasticity by a thermo-sensitive circuit in Drosophila. Sci. Rep. 7:40304. doi: 10.1038/srep40304

Li, M.-T., Cao, L.-H., Xiao, N., Tang, M., Deng, B., Yang, T., et al. (2018). Huborganized parallel circuits of central circadian pacemaker neurons for visual photoentrainment in Drosophila. Nat. Commun. 9:4247. doi: 10.1038/s41467018-06506-5

Liang, X., Ho, M. C., Zhang, Y., Li, Y., Wu, M. N., Holy, T. E., et al. (2019). Morning and evening circadian pacemakers independently drive premotor centers via a specific dopamine relay. Neuron 102, 843-857.e4. doi: 10.1016/j.neuron.2019. 03.028

Liang, X., Holy, T. E., and Taghert, P. H. (2016). Synchronous Drosophila circadian pacemakers display nonsynchronous $\mathrm{Ca} 2+$ rhythms in vivo. Science 351, 976-981. doi: 10.1126/science.aad3997

Majercak, J., Sidote, D., Hardin, P. E., and Edery, I. (1999). How a circadian clock adapts to seasonal decreases in temperature and day length. Neuron 24, 219-230. doi: 10.1016/s0896-6273(00)80834-x

Mezan, S., Feuz, J. D., Deplancke, B., and Kadener, S. (2016). PDF signaling is an integral part of the Drosophila circadian molecular oscillator. Cell Rep. 17, 708-719. doi: 10.1016/j.celrep.2016.09.048

Michael, T. P., Salomé, P. A., and McClung, C. R. (2003). Two arabidopsis circadian oscillators can be distinguished by differential temperature sensitivity. Proc. Natl. Acad. Sci. U.S.A. 100, 6878-6883. doi: 10.1073/pnas.113199 5100

Miyasako, Y., Umezaki, Y., and Tomioka, K. (2007). Separate sets of cerebral clock neurons are responsible for light and temperature entrainment of Drosophila circadian locomotor rhythms. J. Biol. Rhythms 22, 115-126. doi: 10.1177/ 0748730407299344

Moses, K., Ellis, M. C., and Rubin, G. M. (1989). The glass gene encodes a zincfinger protein required by Drosophila photoreceptor cells. Nature 340, 531-536. doi: $10.1038 / 340531 \mathrm{a} 0$

Nash, H. A., Scott, R. L., Lear, B. C., and Allada, R. (2002). An unusual cation channel mediates photic control of locomotion in Drosophila. Curr. Biol. 12, 2152-2158. doi: 10.1016/s0960-9822(02)01358-1

Omoto, J. J., Keleş, M. F., Nguyen, B.-C. M., Bolanos, C., Lovick, J. K., Frye, M. A., et al. (2017). Visual input to the Drosophila central complex by developmentally and functionally distinct neuronal populations. Curr. Biol. 27, 1098-1110. doi: 10.1016/j.cub.2017.02.063

Renn, S. C., Park, J. H., Rosbash, M., Hall, J. C., and Taghert, P. H. (1999). A pdf neuropeptide gene mutation and ablation of PDF neurons each cause severe abnormalities of behavioral circadian rhythms in Drosophila. Cell 99, 791-802. doi: 10.1016/s0092-8674(00)81676-1

Roessingh, S., Rosing, M., Marunova, M., Ogueta, M., George, R., Lamaze, A., et al. (2019). Temperature synchronization of the Drosophila circadian clock protein
PERIOD is controlled by the TRPA channel PYREXIA. Commun. Biol. 2:246. doi: 10.1038/s42003-019-0497-0

Roessingh, S., Wolfgang, W., and Stanewsky, R. (2015). Loss of Drosophila melanogaster TRPA1 function affects "siesta" behavior but not synchronization to temperature cycles. J. Biol. Rhythms 30, 492-505. doi: 10.1177/0748730415605633

Schubert, F. K., Hagedorn, N., Yoshii, T., Helfrich-Förster, C., and Rieger, D. (2018). Neuroanatomical details of the lateral neurons of Drosophila melanogaster support their functional role in the circadian system. J. Comp. Neurol. 526, 1209-1231. doi: 10.1002/cne.24406

Sehadova, H., Glaser, F. T., Gentile, C., Simoni, A., Giesecke, A., Albert, J. T., et al. (2009). Temperature entrainment of Drosophila's circadian clock involves the gene nocte and signaling from peripheral sensory tissues to the brain. Neuron 64, 251-266. doi: 10.1016/j.neuron.2009.08.026

Seluzicki, A., Flourakis, M., Kula-Eversole, E., Zhang, L., Kilman, V., and Allada, R. (2014). Dual PDF signaling pathways reset clocks via TIMELESS and acutely excite target neurons to control circadian behavior. PLoS Biol. 12:e1001810. doi: 10.1371/journal.pbio. 1001810

Shafer, O. T., Kim, D. J., Dunbar-Yaffe, R., Nikolaev, V. O., Lohse, M. J., and Taghert, P. H. (2008). Widespread receptivity to neuropeptide PDF throughout the neuronal circadian clock network of Drosophila revealed by real-time cyclic AMP imaging. Neuron 58, 223-237. doi: 10.1016/j.neuron.2008. 02.018

Shaw, P. J., Cirelli, C., Greenspan, R. J., and Tononi, G. (2000). Correlates of sleep and waking in Drosophila melanogaster. Science 287, 1834-1837. doi: 10.1126/science.287.5459.1834

Stanewsky, R., Kaneko, M., Emery, P., Beretta, B., Wager-Smith, K., Kay, S. A., et al. (1998). The cryb mutation identifies cryptochrome as a circadian photoreceptor in Drosophila. Cell 95, 681-692. doi: 10.1016/s0092-8674(00)81 638-4

Stoleru, D., Peng, Y., Agosto, J., and Rosbash, M. (2004). Coupled oscillators control morning and evening locomotor behaviour of Drosophila. Nature 431, 862-868. doi: 10.1038/nature02926

Stoleru, D., Peng, Y., Nawathean, P., and Rosbash, M. (2005). A resetting signal between Drosophila pacemakers synchronizes morning and evening activity. Nature 438, 238-242. doi: 10.1038/nature04192

Sun, Y., Nern, A., Franconville, R., Dana, H., Schreiter, E. R., and Looger, L. L. (2017). Neural signatures of dynamic stimulus selection in Drosophila. Nat. Neurosci. 20, 1104-1113. doi: 10.1038/nn.4581

Tanoue, S., Krishnan, P., Krishnan, B., Dryer, S. E., and Hardin, P. E. (2004). Circadian clocks in antennal neurons are necessary and sufficient for olfaction rhythms in Drosophila. Curr. Biol. 14, 638-649. doi: 10.1016/j.cub.2004.04.009

Tataroglu, O., and Emery, P. (2015). The molecular ticks of the Drosophila circadian clock. Curr. Opin. Insect Sci. 7, 51-57. doi: 10.1016/j.cois.2015.01.002

Timaeus, L., Geid, L., and Hummel, T. (2017). A topographic visual pathway into the central brain of Drosophila. bioRxiv [preprint]. doi: 10.1101/18 3707

Van Alphen, B., Yap, M. H., Kirszenblat, L., Kottler, B., and van Swinderen, B. (2013). A dynamic deep sleep stage in Drosophila. J. Neurosci. 33, 6917-6927. doi: 10.1523/JNEUROSCI.0061-13.2013

van der Vinne, V., Riede, S. J., Gorter, J. A., Eijer, W. G., Sellix, M. T., and Menaker, M. (2014). Cold and hunger induce diurnality in a nocturnal mammal. Proc. Natl. Acad. Sci. U.S.A. 111, 15256-15260. doi: 10.1073/pnas.141313 5111

Veleri, S., Brandes, C., Helfrich-Förster, C., Hall, J. C., and Stanewsky, R. (2003). A self-sustaining, light-entrainable circadian oscillator in the Drosophila brain. Curr. Biol. 13, 1758-1767. doi: 10.1016/j.cub.2003.09.030

Wheeler, D. A., Hamblen-Coyle, M. J., Dushay, M. S., and Hall, J. C. (1993). Behavior in light-dark cycles of Drosophila mutants that are arrhythmic, blind, or both. J. Biol. Rhythms 8, 67-94. doi: 10.1177/07487304930080 0106

Wolfgang, W., Simoni, A., Gentile, C., and Stanewsky, R. (2013). The Pyrexia transient receptor potential channel mediates circadian clock synchronization to low temperature cycles in Drosophila melanogaster. Proc. Biol. Sci. 280:20130959. doi: 10.1098/rspb.2013.0959

Yadlapalli, S., Jiang, C., Bahle, A., Reddy, P., Meyhofer, E., and Shafer, O. T. (2018). Circadian clock neurons constantly monitor environmental temperature to set sleep timing. Nature 555, 98-102. doi: 10.1038/nature25740 
Yoshii, T., Hermann, C., and Helfrich-Förster, C. (2010). Cryptochrome-positive and-negative clock neurons in Drosophila entrain differentially to light and temperature. J. Biol. Rhythms 25, 387-398. doi: 10.1177/074873041038 1962

Yoshii, T., Heshiki, Y., Ibuki-Ishibashi, T., Matsumoto, A., Tanimura, T., and Tomioka, K. (2005). Temperature cycles drive Drosophila circadian oscillation in constant light that otherwise induces behavioural arrhythmicity. Eur. J. Neurosci. 22, 1176-1184. doi: 10.1111/j.1460-9568.2005.04295.x

Yoshii, T., Wülbeck, C., Sehadova, H., Veleri, S., Bichler, D., Stanewsky, R., et al. (2009). The neuropeptide pigment-dispersing factor adjusts period and phase of Drosophila's clock. J. Neurosci. 29, 2597-2610. doi: 10.1523/JNEUROSCI.543908.2009

Young, J., and Armstrong, J. (2010). Structure of the adult central complex in Drosophila: organization of distinct neuronal subsets. J. Comp. Neurol. 518, 1500-1524. doi: 10.1002/cne.22284

Zhang, L., Chung, B. Y., Lear, B. C., Kilman, V. L., Liu, Y., and Mahesh, G. (2010). DN1p circadian neurons coordinate acute light and PDF inputs to produce robust daily behavior in Drosophila. Curr. Biol. 20, 591-599. doi: 10.1016/j.cub. 2010.02.056

Zhang, Y., Liu, Y., Bilodeau-Wentworth, D., Hardin, P. E., and Emery, P. (2010). Light and temperature control the contribution of specific DN1 neurons to Drosophila circadian behavior. Curr. Biol. 20, 600-605. doi: 10.1016/j.cub.2010. 02.044

Conflict of Interest: The authors declare that the research was conducted in the absence of any commercial or financial relationships that could be construed as a potential conflict of interest.

Copyright (c) 2020 Lamaze and Stanewsky. This is an open-access article distributed under the terms of the Creative Commons Attribution License (CC BY). The use, distribution or reproduction in other forums is permitted, provided the original author(s) and the copyright owner(s) are credited and that the original publication in this journal is cited, in accordance with accepted academic practice. No use, distribution or reproduction is permitted which does not comply with these terms. 